\title{
Espinha bífida em neonato felino: relato de caso
}

\section{Spina bifida in newborn cat: case report}

\author{
Dayane Francisca Higino Miranda ${ }^{1}$; Maíra Soares Ferraz ${ }^{2 *}$; José Wilson Costa \\ Azevedo Júnior ${ }^{3}$; Eunice Anita Moura Fortes ${ }^{4}$; Aírton Mendes Conde Júnior ${ }^{5}$; \\ Maria Acelina Martins Carvalho ${ }^{6}$
}

\section{Resumo}

Este trabalho tem como objetivo descrever os aspectos macroscópicos da doença e os achados radiológicos relacionados à espinha bífida em um neonato felino. Uma fêmea felina foi atendida em trabalho de parto numa clínica veterinária em Teresina-PI. Ao realizar o exame ultrassonográfico foi possível detectar a presença de três fetos com idade aproximada de 62 dias e com batimentos cardíacos diminuídos. A paciente foi submetida imediatamente à cirurgia cesariana com ovariossalpingohisterectomia. Um dos neonatos apresentava alterações compatíveis com espinha bífida. A proprietária optou pela eutanásia do neonato por não se conhecer um tratamento eficaz para o caso. Ainda são escassas as pesquisas sobre o desenvolvimento embrionário voltadas para prevenção e tratamento dessas anomalias. Estas pesquisas são necessárias a fim de garantir um bom prognóstico aos pacientes portadores.

Palavras-chave: Anomalias vertebrais, gatos, malformações congênitas

\begin{abstract}
This paper aims to describe the macroscopic aspects of disease and radiological findings related to spina bifida in a newborn cat. A female cat was seen in labor at a veterinary clinic in Teresina-PI. By performing the ultrasound test it was possible detect the presence of three fetuses aged approximately 62 days and decreased heart rate. The patient underwent surgery immediately to cesarean section with ovariohysterectomy. One of the newborn cat showed changes consistent with spina bifida. The owner opted for the newborn cat euthanasia because is not known effective treatment for the case. There are insufficient researchs on the embryonic development aimed at prevention and treatment of these anomalies. These studies are necessary to ensure a good prognosis for patients.
\end{abstract}

Key words: Vertebral anomalies, cat, congenital malformation

\section{Introdução}

As anomalias envolvendo os arcos vertebrais são denominadas coletivamente de espinha bífida, denotando a falta de fusão das metades embrionárias dos arcos vertebrais. Nas alterações graves, a medula espinhal e as meninges também estão envolvidas. A espinha bífida compreende desde tipos clinicamente significativos a pequenas anomalias sem importância (MOORE; PERSAUD, 2008). Apesar da patogênese embrionária dessa desordem não estar totalmente

\footnotetext{
1 Médica Veterinária residente da Universidade Federal do Piauí, HVU/UFPI, Teresina, PI. E-mail: dayaneprincesa@gmail.com

2 Médica Veterinária doutoranda em Ciência Animal pela UFPI, Teresina, PI. E-mail: mairasferraz@yahoo.com.br

3 Discente de graduação em Medicina Veterinária pela UFPI, Teresina, PI. E-mail: jwsafadao02@hotmail.com

4 Médica Veterinária, Prof ${ }^{a}$ do Dept ${ }^{0}$ de Morfologia/CCS/UFPI, Teresina, PI. E-mail: euniceamf@ufpi.br

5 Médico Veterinário, Prof. do Dept ${ }^{\circ}$ de Morfologia/CCS/UFPI, Teresina, PI. E-mail: airtoncondejr@uol.com.br

${ }^{6}$ Médica Veterinária, Prof ${ }^{a}$ do Dept ${ }^{\circ}$ de Morfofisiologia Veterinária/CCA/UFPI, Teresina, PI. E-mail: mcelina@ufpi.edu.br

* Autor para correspondência 
elucidada (BRAUND, 1994), a hiperplasia das células do tubo dorsal que afeta a fusão do tubo neural e dos arcos vertebrais e/ou um defeito vascular que limita ou impede o fluxo sanguíneo para a região dorsal da coluna vertebral têm sido sugeridos como possíveis causas (BRAUND, 1994). Esta condição tem sido descrita em cães, gatos, bovinos e também em leões (BORGES et al., 1997; GALLOWAY et al., 2002; MATTOS JÚNIOR et al., 2007; CAMPOS et al., 2009). Entretanto, uma incidência relativamente alta de espinha bifida é associada a cães jovens da raça Bullldog Inglês e em gatos da raça Manx (WALKER, 2002).

Clinicamente, a doença é reconhecida por afetar a deambulação dos animais, podendo apresentar ataxia e paresia dos membros pélvicos, incontinência fecal e urinária, analgesia perineal e esfíncter anal (BRAUND, 1994). O diagnóstico é baseado na apresentação macroscópica associada a radiografias simples, mielografias e tomografia computadorizada (BRAUND, 1994; WALKER, 2002). Em humanos, o envolvimento de nervos pode afetar os membros inferiores, sendo o pé torto congênito um achado freqüente em fetos portadores de espinha bífida aberta (HISABA et al., 2003).

Em gatos, as principais afecções do desenvolvimento incluem as malformações congênitas como a disgenesia sacrococcígea, luxação e sub-luxação atlanto-occipital, hemivértebra, estenose lombossacral e o grupo das mielodisplasias (BRAUND, 1994; TUDURY et al., 2000). Outros estados disráficos que envolvem defeitos vertebrais e de medula espinhal, como espinha bífida e "sinus dermóide", também acometem os gatos (BRAUND, 1994).

Apesar das publicações atuais sobre malformações congênitas em felinos (ARAÚJO et al., 2009), relatos de espinha bífida em gatos não têm sido publicados recentemente. Portanto, este trabalho tem como objetivo descrever os aspectos macroscópicos e os achados radiológicos relacionados à espinha bífida em um neonato felino.

\section{Relato de Caso}

Em Agosto de 2008, foi atendido na clínica veterinária Animal's, em Teresina-PI, um felino do sexo feminino com histórico de estro cerca de 60 dias antes da consulta. Ao observar os sinais do estro a proprietária administrou anticoncepcional injetável (Acetato de Medroxiprogesterona) por via subcutânea. Cerca 24 horas antes da consulta a paciente começou a apresentar secreção vulvar escura, entretanto não demonstrou sinais de contrações musculares para o parto. Segundo a proprietária a paciente estava apática e dependente. Relata também diminuição de apetite um dia antes do início da secreção. A urina e as fezes estavam normais segundo a proprietária.

Ao exame clínico a paciente apresentavase apática, com mucosas normocoradas, e sem alterações nos linfonodos. Não havia sinais aparentes de desidratação. À palpação abdominal foi possível detectar a presença mais de um feto e também movimentação fetal. A vulva apresentava secreção sanguinolenta. As glândulas mamárias apresentavam discreto aumento de volume com pouca secreção láctea. A paciente foi encaminhada para a realização de exame ultrassonográfico em que foi possível diagnosticar a presença de pelo menos três fetos de idade aproximada de 62 dias apresentando batimentos cardíacos diminuídos, indicando sofrimento fetal, além de pequena quantidade de líquidos fetais. Devido ao caráter emergencial do caso, a paciente foi submetida à cirurgia cesariana com ovariossalpingohisterctomia (a pedido da proprietária). A paciente foi submetida à seguinte medicação pré-anéstesica: acepromazina $(0,05$ $\mathrm{mg} / \mathrm{kg}$ ) associada a meperidina (3 $\mathrm{mg} / \mathrm{kg}$ ) na mesma seringa aplicados por via intramuscular. Nesse momento foram administradas, por via 
intramuscular, as medicações antinflamatória (meloxicam - 0,1 mg/kg) e antibiótica (penicilina $\mathrm{G}$ benzatina - $40.000 \mathrm{UI} / \mathrm{kg}$ ). Após 10 minutos, a indução anestésica foi realizada com cloridrato de quetamina $(2,5 \mathrm{mg} / \mathrm{kg})$ e midazolan $(0,2 \mathrm{mg} / \mathrm{kg})$ associados na mesma seringa e aplicados por via endovenosa. A manutenção da anestesia foi feita com isofluorano em circuito semi-fechado. Foi realizada primeiramente a cesariana e três fetos foram retirados com vida, porém debilitados. Um dos fetos apresentava uma malformação nos membros posteriores e na região torácica distal e lombassacra da coluna vertebral com ausência de pele nesse local. Após a cesariana foi realizada a OSH da paciente. A paciente recebeu alta médica juntamente com os outros fetos no mesmo dia após recuperação anestésica.

Por se tratar de uma alteração congênita com conseqüências que comprometeriam a qualidade de vida do animal, e por não se conhecer um tratamento eficaz para a espinha bífida em medicina veterinária, a proprietária optou pela eutanásia do recém-nascido, que foi realizada com a sobredose anestésica (quetamina + xilazina), aplicados por via intraperitoneal.

Após a eutanásia do neonato, o mesmo foi radiografado, fotografado e fixado em formol a $10 \%$ para posterior descrição e dissecação anatômica. $\mathrm{O}$ feto felino apresentava pêlos distribuídos por todo o corpo com exceção da região torácica distal, lombar e sacral da coluna vertebral. Foi possível observar uma fenda na região torácica distal e lombar dorsal da coluna vertebral, com ausência de pele na região. As vértebras T13 a L3 apresentam seus processos dorsais não fusionados. Esta fenda resultou na exposição e protrusão da medula espinhal e das meninges. Os membros posteriores também apresentavam deformações. O neonato estava totalmente formado tendo um comprimento total de $15,8 \mathrm{~cm}$, circunferência torácica de $7,8 \mathrm{~cm}$, CRL de $10,2 \mathrm{~cm}$ e a alteração na coluna vertebral medindo 5,16cm (Figura 1).
Figura 1. Fotografia mostrando aspecto do neonato felino com espinha bífida ao nascimento (A) e após fixação com formol 10\% e dissecação (B).

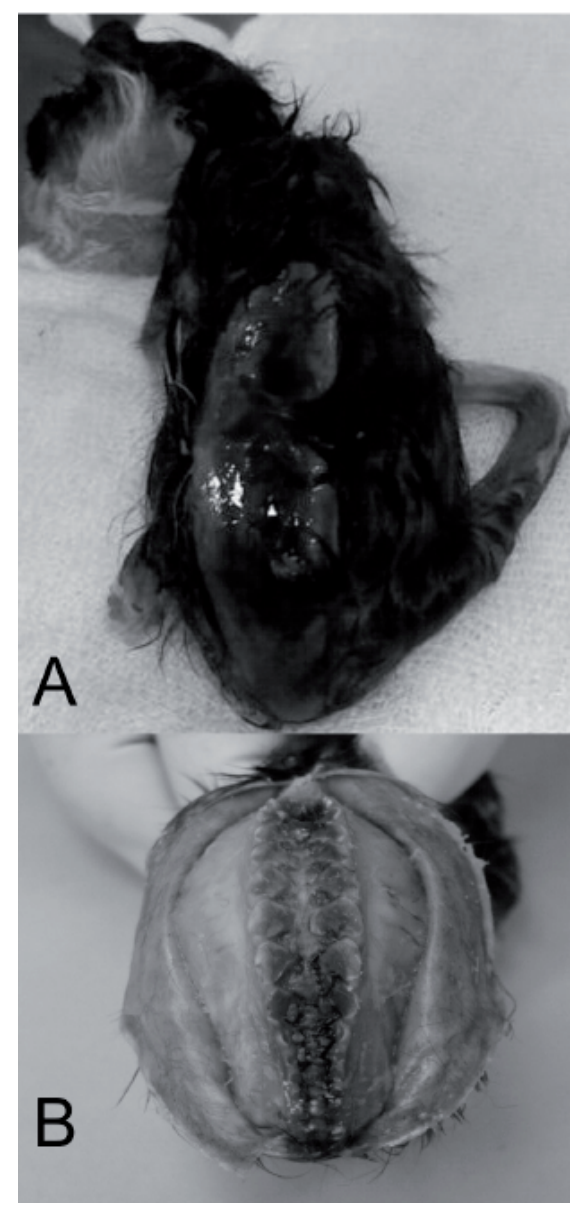

As radiografias ventro-dorsais da coluna vertebral revelaram um defeito na fusão dos processos espinhosos dorsais das vértebras torácicas distais e lombares (T13- L3). As radiografias látero-laterais revelaram xifose de toda a região lombar da coluna vertebral. Foi possível observar que todos os outros órgãos e sistemas estavam normais (Figura 2).

O diagnóstico de espinha bífida foi baseado na observação macroscópica da fissura, formada por fusão incompleta dos processos espinhosos dorsais, e confirmado por achados radiográficos coerentes com esta anormalidade segundo Braund (1994) e Walker (2002), apesar da mielografia e da tomografia computadorizada. Neste caso, a alteração ocorreu na região torácica distal e lombar. 
Figura 2. Fotografia dos exames radiográficos nas posições dorso-ventral (A) e látero-lateral (B) evidenciando alterações ósseas nas vértebras (T13 a S3) e nos membros posteriores.

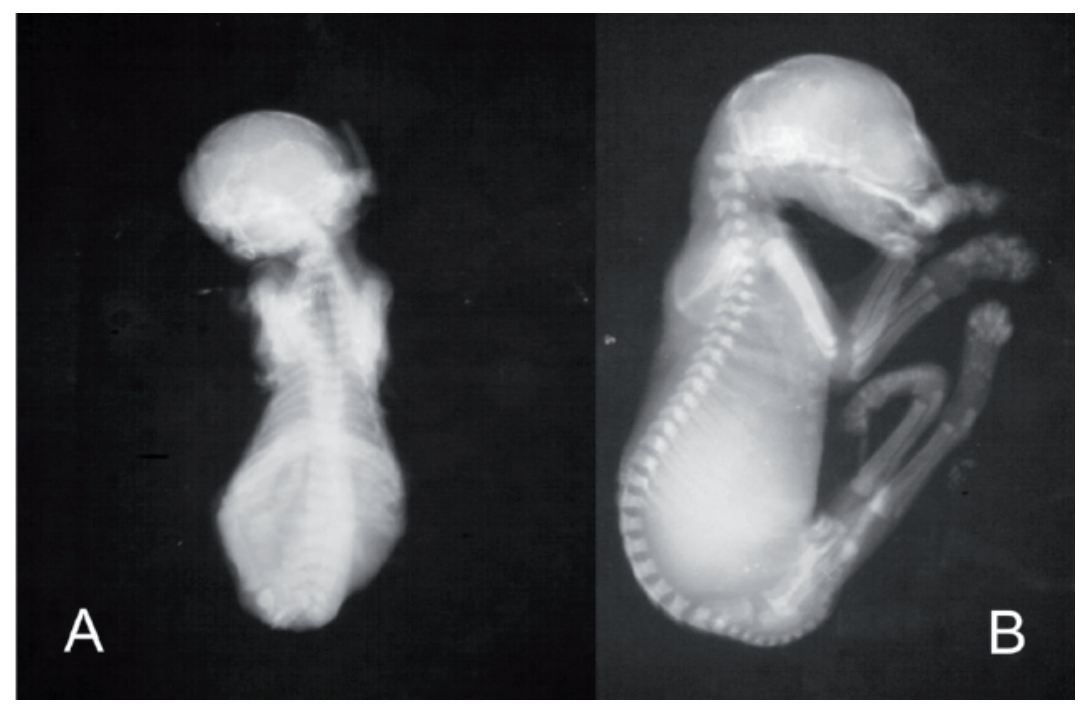

Entretanto, a fissura pode envolver a maior parte do arco vertebral ou apenas o processo espinhoso dorsal (BRAUND, 1994), podendo ser observada na região cervical ou torácica dos vertebrados (KEALY; McALLISTER, 2000).

Em medicina veterinária não é conhecido nenhum tratamento eficaz para a espinha bífida e o prognóstico pode variar (BRAUND, 1994). Uma equipe realizou uma intervenção cirúrgica em um cão com lesões semelhantes, e acompanhamentos posteriores indicaram que o cão recuperou-se da malformação espinhal, mas persistiu o transtorno urinário e incontinência fecal, comuns nesse tipo de malformação (SHAMIR; ROCHKIND; JOHNSTON, 2001). Diante da situação, a proprietária optou pela eutanásia do neonato.

$\mathrm{Na}$ medicina humana espinha bífida é um grave problema de saúde pública e tratada por prevenção. A frequência deste defeito neural nos E.U.A. foi reduzida devido à ingestão diária de 400 microgramas de ácido fólico antes ou durante a gravidez, e que resultou em níveis ainda mais baixos de nascimentos, óbitos fetais rescisões e eletivas (MERSEREAU et al., 2004). Entretanto, ainda são escassas as pesquisas sobre o desenvolvimento embrionário voltadas para prevenção e tratamento dessas anomalias em animais domésticos.

Portanto, pesquisas devem ser realizadas para avaliar o efeito da administração de ácido fólico para fêmeas domésticas gestantes a fim de prevenir a ocorrência do defeito, bem como, para garantir um bom prognóstico aos pacientes portadores.

\section{Referências}

ARAÚJO, F. P.; ARAÚJO, B. M.; KEMPER, B.; TUDURY, E. A. Associação de agenesia sacrococcígea e atresia anal em gato sem raça definida. Ciência Rural, Santa Maria, v. 39, n. 6, p. 1893-1896, 2009.

BORGES, A. S.; MENDES, L. C. N.; VASCONCELOS, R.; ALVES, A. L. G.; RODRIGUES, C. A. Espinha bífida e mielodisplasia em ruminantes: relato de caso. Arquivo Brasileiro de Medicina Veterinária e Zootecnia, Belo Horizonte, v. 49, n. 6, p. 685-692, 1997.

BRAUND, K .G. Clinical syndromes in veterinary neurology. 2. ed. St. Louis: Mosby, 1994. 476 p.

CAMPOS, K. F.; SOUSA, M. G. S.; SILVA, N. S.; OLIVEIRA, C. H. S.; DUARTE, M. D.; BARBOSA, J. D.; OLIVEIRA, C. M. C. Doenças congênitas em bovinos diagnosticadas pela central de diagnóstico veterinário (CEDIVET) da Universidade Federal do Pará, no período de 1999 a 2009. Ciência Animal Brasileira, Goiânia, v. 1, 2009, p. 13-18. Suplemento 1. 
GALLOWAY, D. S.; COKE, R. L.; ROCHAT, M. C.; RADINSKY, M. A.; HOOVER, J. P.; CARPENTER, J. W.; HUBBARD, J. J.; KETZ-RILEY, C. J. Spinal compression due to atlantal vertebral malformation in two African lions (Pantera leo). Journal of Zoo and Wildlife Medicine, Lawrence, v. 33, n. 3, p. 249-255, 2002.

HISABA, W. J.; MORON, A. F.; CAVAHLEIRO, S.; SANTANA, R. M.; PASSOS, J. P.; CORDIOLI, E. Espinha bífida aberta: achados ultra- sonográficos e presença de contrações uterinas na predição da evolução motora neonatal. Revista Brasileira de Ginecologia e Obstetrícia, Rio de Janeiro, v. 25, n. 6, p. 425-430, 2003.

KEALY, J. K.; McALLISTER, H. The skull and vertebral column. In: KEALY, J. K.; McALLISTER, H. (Ed.). Diagnostic radiology and ultrasonography of the dog and cat. 3. ed. Philadelphia: W.B. Saunders, 2000. p. 387.

MATTOS JÚNIOR, E.; RIBEIRO, E. A.; SANTOS, G. J. von G.; RUSSO, C.; HEADLEY, S. A. Spina bifida in a dead stillbirth mongrel dog. Semina: Ciências Agrárias, Londrina, v. 28, n. 2, p. 295-298, 2007.

MERSEREAU, P.; KILKER, K.; CARTER, H.; FASSET, E.; WILLIAMS, J.; FLORES, A.; PRUE, C.; WILLIAMS, L.; MAI, C.; MULINARE, J. Spina bifida and anencephaly before and after folic acid mandate United States, 1995- 1996 and 1999-2000. Journal of the American Medical Association, Chicago, v. 292, n. 3, p. 325-326, 2004.

MOORE, K. L.; PERSAUD, T. V. N. Embriologia clínica. 2. ed. Rio de Janeiro: Guanabara Koogan, 2008.

SHAMIR, M.; ROCHKIND, S.; JOHNSTON, D. Surgical treatment of tethered spinal cord syndrome in a dog with myelomeningocele. The Veterinary Record, London, v. 148, n. 1, p. 755-756, 2001.

TUDURY. E. A.; CAMARGO, P. L.; GRAÇA, D. L.; PEDRO NETO, O. Mielodisplasia segmentar múltipla em gato. Ciência Rural, Santa Maria, v. 30, n. 3, p. 529531, 2000.

WALKER, M. A. The vertebrae - canine and feline. In: THRALL, D. E. (Ed.). Textbook of veterinary diagnostic radiology. 4. ed. Philadelphia: W.B. Saunders, 2002. p. 98-109. 
